\title{
Congenital heart disease in Down's syndrome: two year prospective early screening study
}

\author{
T R J Tubman, M D Shields, B G Craig, H C Mulholland, N C Nevin
}

\begin{abstract}
Objective-To determine the effectiveness of clinical examination, chest radiography, and electrocardiography compared with echocardiography in detecting congenital heart disease early in the life of children with Down's syndrome.
\end{abstract}

Design-Prospective two year screening survey.

Setting-Regional paediatric cardiology service, Northern Ireland.

Patients - 81 newborn infants with Down's syndrome born in Northern Ireland between November 1987 and November 1989.

Interventions - Clinical examination, chest radiography, and electrocardiography soon after birth followed by cross sectional Doppler echocardiography.

Main outcome measures-Diagnostic ability of clinical examination, radiography, and electrocardiography compared with echocardiographic findings.

Results -34 babies had congenital heart disease detected by echocardiography (13 had atrioventricular septal defects, seven secundum atrial septal defects, six a solitary patent ductus arteriosus, five isolated ventricular septal defects, and three combinations of heart defects). Individual examination methods were insensitive (the sensitivity of clinical examination was 0.53 , of radiography 0.44 , and of electrocardiography 0.41 ) but highly specific (the specificity of clinical examination was 0.94 , of radiography 0.98 , and of electrocardiography 1.0 ), although sensitivity improved when the three techniques were combined (the sensitivity was 0.71 , the specificity 0.91).

Conclusion-Echocardiography performed early in life can detect congenital heart disease that might otherwise be missed. Early detection may help prevent complications such as pulmonary vasi:ular disease that may adversely affect the outcome of cardiac surgery.

\section{Introduction}

Down's syndrome (trisomy 21) is the commonest cause of mental handicap in Northern Ireland. ${ }^{1}$ Its association with congenital heart disease is well known, the reported incidence being $40-60 \% .^{2-4}$ Congenital heart disease contributes significantly to the morbidity and mortality of children with Down's syndrome, who may develop congestive heart failure, pulmonary vascular disease, pneumonia, or failure to thrive. In the first few days of life symptoms or signs may be absent or minimal despite the presence of significant congenital heart disease. For some time we have thought that parents of some children with Down's syndrome and congenital heart disease (particularly atrioventricular septal defects) have been reassured initially by paediatricians that their child's heart is probably normal until sequelae such as pulmonary vascular disease have become evident. We therefore undertook a prospective study to screen for congenital heart disease in babies with Down's syndrome at or as soon after birth as possible. We determined the incidence of congenital heart disease in such babies born in Northern Ireland and the incidences of specific forms of cardiac defect. We also compared the effectiveness of the current practice of clinical examination, chest radiography, and electrocardiography with screening by cross sectional echocardiography.

\section{Patients and methods}

We prospectively examined all new babies with Down's syndrome who were born in Northern Ireland within a two year period from November 1987 to November 1989. The diagnosis in all babies was later confirmed by chromosomal analysis. All consultant paediatricians and obstetricians in Northern Ireland were asked to notify the Regional Paediatric Cardiology Service of new cases of Down's syndrome at or as soon after birth as possible. Clinical findings suggestive of congenital heart disease including cyanosis, tachypnoea, an abnormal precordial impulse, or a murmur were recorded by the referring clinician; chest radiography and electrocardiography were performed when possible.

Babies were then referred to the regional paediatric cardiology unit for further clinical examination and cross sectional Doppler echocardiography. If a chest radiograph or electrocardiogram had not been taken by the referring hospital the salient procedure was then performed. In several cases (mainly because of geographical distance) echocardiography could not be performed within the first few days of life; under these circumstances babies were seen at one of four paediatric cardiology clinics held monthly in peripheral district general hospitals. Thus newborn babies with Down's syndrome could be seen within the first month of life and in most cases within the first few days of life for a full cardiological assessment.

In addition, monthly returns of every new case of Down's syndrome confirmed by chromosomal analysis were supplied by the Northern Ireland Genetics Service. These were cross checked against clinical referrals; the parents or general practitioners of missing babies were contacted by telephone and arrangements made to examine these babies as soon as possible. In general, babies born in hospitals with no on site paediatric service were more likely to be picked up in this way.

Cross sectional Doppler echocardiography was taken as the "gold standard" for detecting congenital heart defects and the results from it were compared with those from standard methods of examination - that is, clinical examination, chest radiography, and electrocardiography - both separately and collectively. In a few babies echocardiography detected a small patent
Dr T R J Tubman, Neonatal

Hospital, Belfast BT12 6BB.

BMF 1991;302:1425-7 
ductus arteriosus or a patent foramen ovale within the first few days of life. As these findings could have been a part of the normal neonatal transitional circulation, reassessment was made one month later for a definitive diagnosis.

Data were analysed to determine sensitivity, specificity, and positive and negative predictive values ${ }^{5}$ for each of the clinical methods of screening used alone and in combination and were compared with the results for echocardiography.

\section{Results}

INCIDENCE OF CONGENITAL HEART DISEASE

In the two year study period a total of 81 babies were referred either by clinicians (69) or by the genetics service (12) and screened for congenital heart disease. Fifty three were boys and 28 girls. There were 53847 live births in Nothern Ireland during this period, giving an annual incidence of 1.50 per 1000 live births. Sixty one babies were examined by echocardiography in the first two weeks of life and 74 before 3 months of age.

Thirty four babies had congenital heart disease detectable by cross sectional echocardiography. The proportion of affected boys and girls was similar $(40 \%$ males $v 46 \%$ females). Of the affected babies, 13 had atrioventricular septal defects, seven secundum atrial septal defects, six a large solitary patent ductus arteriosus, and five isolated ventricular septal defects. Three babies had a combination of heart defectsnamely, atrioventricular septal defect with coarctation of aorta and patent ductus arteriosus; secundum atrial septal defect with ventricular septal defect and a right sided overriding aorta (but no pulmonary stenosis); and ventricular septal defect with secundum atrial septal defect and mild pulmonary valvar stenosis. Eight of the 13 atrioventricular septal defects had an associated patent ductus arteriosus, usually with detectable left to right flow. Similarly, three of the seven secundum atrial septal defects had an associated patent ductus arteriosus.

\section{SCREENING}

Table I shows the diagnostic ability of the three clinical tests alone and in combination.

Clinical examination-Of the 81 babies examined, 21 had abnormal clinical findings suggestive of congenital heart disease. Heart disease was confirmed in 18 of them, but the remaining three had structurally normal hearts (two had "cyanosis" due to polycythaemia and persistent fetal circulation and one had an innocent systolic murmur). Of the 60 babies thought by referring clinicians to have no heart disease, 16 had structural defects detected by echocardiography (five had atrioventricular septal defects, six secundum atrial septal defects, three ventricular septal defects, one had a patent ductus arteriosus, and one a combination of defects). Five of these babies had more subtle clinical signs, such as an accentuated pulmonary second sound, detected on re-examination by cardiologists (two had atrioventricular septal defects, one secundum atrial septal defect, one a patent ductus arteriosus, and one a

TABLE I - Positive and negative diagnoses of heart disease in 81 babies with Down's syndrome by clinical examination, chest radiography, electrocardiography, and their combination compared with results of echocardiography. Values are numbers of cases

\begin{tabular}{|c|c|c|c|c|c|c|c|c|}
\hline & \multicolumn{2}{|c|}{ Clinical examination } & \multicolumn{2}{|c|}{ Chest radiography } & \multicolumn{2}{|c|}{ Electrocardiography } & \multicolumn{2}{|c|}{ Three combined } \\
\hline & Positive & Negative & Positive & Negative & Positive & Negative & Positive & Negative \\
\hline \multicolumn{9}{|c|}{ Echocardiography: } \\
\hline Negative & 3 & 44 & 1 & 46 & 0 & 47 & 4 & 43 \\
\hline Positive & 18 & 16 & 15 & 19 & 13 & 19 & 24 & 10 \\
\hline
\end{tabular}

*Not performed in two cases. combination of defects). Thus clinical examination by referring clinicians had a sensitivity of 0.53 and a specificity of 0.94 .

Chest radiography-Sixteen babies had an initial chest radiograph reported as abnormal; on reexamination one of these was suggestive of transient tachypnoea of the newborn rather than pulmonary oedema. Nineteen babies had a normal chest radiograph but had a structural abnormality diagnosed by echocardiography (three had atrioventricular septal defects, five ventricular septal defects, six had secundum atrial septal defects, four a patent ductus arteriosus, and one a combination of defects). Compared with echocardiography, chest radiography had a sensitivity of 0.44 and a specificity of 0.98 .

Electrocardiography-Seventy nine of the 81 babies were examined by electrocardiography. In one baby the heart condition had already been diagnosed by echocardiography; the other baby, a preterm of 28 weeks' gestation, died before electrocardiography could be performed but had a chest radiograph and an echocardiogram. Both babies had congenital heart disease (one had an atrioventricular septal defect, the other a secundum atrial septal defect). Sixty six babies had a normal electrocardiogram, but 19 of them had congenital heart disease (two had atrioventricular septal defects, four ventricular septal defects, five secundum atrial septal defects, five a patent ductus arteriosus, and three a combination of defects). Of the 13 babies with an abnormal electrocardiogram - that is, an abnormal QRS axis or evidence of ventricular hypertrophy - 10 had atrioventricular septal defects, one a ventricular septal defect, one a secundum atrial septal defect, and one a large patent ductus arteriosus. There were no false positive electrocardiograms. Thus electrocardiography alone had a much higher specificity and positive predictive value (both $1 \cdot 0$ ) than the two other methods of screening.

The three techniques combined-When the three diagnostic procedures were combined, with at least one of them giving a positive result, sensitivity was greater than when any one test was used alone, though specificity was reduced because of four false positive results (table II). Despite the increased sensitivity, 10 babies had heart disease which would not have been detected without echocardiography (two had atrioventricular septal defects, five secundum atrial septal defects, two ventricular septal defects, and one a patent ductus arteriosus)

TABLE II - Sensitivity, specificity, and positive and negative predictive values of clinical examination, chest radiography, electrocardiography, and the three techniques combined

\begin{tabular}{lcccc}
\hline & & & \multicolumn{2}{c}{ Predictive value } \\
\cline { 4 - 5 } Diagnostic test & Sensitivity & Specificity & Positive & Negative \\
\hline Clinical examination & 0.53 & 0.94 & 0.86 & 0.73 \\
Chest radiography & $0 \cdot 44$ & 0.98 & 0.94 & 0.71 \\
Electrocardiography & 0.41 & 1.0 & $1 \cdot 0$ & 0.71 \\
Three techniques combined & 0.71 & 0.91 & 0.86 & 0.81
\end{tabular}

$\star$ At least one technique giving a positive result.

\section{Discussion}

The incidence of Down's syndrome for the two years of the study was 1.50 per 1000 live births (or one in 670 ). This is in keeping with that generally quoted with our experience in Northern Ireland: the average annual incidence for $1980-9$ was 1.47 per 1000 live births $(95 \%$ confidence interval $1 \cdot 38$ to $1 \cdot 56)$. The incidences of congenital heart disease and of the three major types of cardiac defects were similar to those found by some workers ${ }^{23}$ but different from those found by others. These differences reflect the nature of previous studies. Few prospective studies of congenital heart disease in 
Down's syndrome have been performed'; most have been retrospective and some have included only children with known cardiac disease examined at cardiac catheterisation ${ }^{+6}$ or at necropsy. ${ }^{67}$ We studied all babies with Down's syndrome irrespective of whether they had symptoms or signs. Cross sectional Doppler echocardiography in a logical sequential approach can accurately detect most cardiac defects ${ }^{8}$; to date none of the babies in our study who had echocardiographically normal hearts have been referred back because of cardiac problems. Our results therefore probably reflect the true incidence of congenital heart disease in the population studied.

Screening for congenital heart disease by clinical examination, chest radiography, electrocardiography, or a combination of all three of these techniques may fail to detect clinically important defects in the neonatal period. The sensitivity of each of the techniques alone was low, though it was improved when they were used in combination. This insensitivity might be due because symptoms are not present and clinical and radiological signs of a left to right shunt may not have developed in the immediate postnatal period owing to the high pulmonary vascular resistance at this time. ${ }^{2}$ Electrocardiograms may be abnormal, particularly in the presence of an atrioventricular septal defect ${ }^{2}$; we found that an abnormal electrocardiogram had a high positive predictive value for congenital heart disease. In some units neonatal electrocardiography may not be readily available or the results incorrectly interpreted because of unfamiliarity with normal findings in the newborn. Diagnosis is rapid, non-invasive, and definitive by echocardiography. It is best performed under the direction of paediatric cardiologists, who have the necessary equipment, technical skill, and clinical experience. The introduction of screening of all babies with Down's syndrome should not result in a major increase in workload for individual paediatric cardiologists; in Northern Ireland this would mean less than one extra new patient a week.

Pulmonary vascular disease is more common and occurs at an earlier stage in babies with Down's syndrome and congenital heart disease, ${ }^{9}$ particularly in those with large left to right shunts. ${ }^{910}$ Such babies have a higher pulmonary artery pressure both at cardiac catheterisation ${ }^{10}$ and preoperatively than have normal babies." About $30-40 \%$ of children have irreversible changes precluding surgery at the time of presentation. ${ }^{10} 11$ Whether these babies should be offered surgery has been a subject of some debate. ${ }^{12-14}$ In Northern Ireland the policy is to offer the full range of treatment to such children, unless the treatment is contraindicated or not desired by their parents. In some centres surgery is not routinely offered as the actuarial survival without treatment is thought to be similar to or better than than that of children treated surgically. ${ }^{12}$ Recent evidence suggests, however, that the actuarial survival of babies who have had surgery for atrioventricular septal defects is much better than of those treated by medical means alone ${ }^{15}$; most of these babies have Down's syndrome. This is partly related to improvements in surgical skill, though the time of surgical intervention is also critical; operation performed after the first year of life has twice the mortality of surgery before the first year, ${ }^{15}$ mainly because pulmonary vascular changes develop after only six months of life in Down's syndrome. ${ }^{16}$ In this study we did not determine whether earlier detection and subsequent management of congenital heart disease had any effect on morbidity or outcome. This is an important but peripheral issue that can be investigated only by a randomised controlled trial, if such a trial is ethically justified.

Many parents of babies with Down's syndrome are aware of the increased risk of congenital heart disease and would be reassured by a normal echocardiogram. When it is abnormal parents can be informed at an early stage and counselled appropriately. Early diagnosis of congenital heart disease, particularly of large left to right shunts, could enable a paediatrician to follow the baby carefully, to start medical treatment with diuretics and digoxin at an earlier stage, and possibly to plan for earlier surgical intervention should this be indicated. We believe that babies with Down's syndrome should be screened for congenital heart disease in units where surgery, when needed, is a normal treatment option. Screening should preferably be by echocardiography as this is highly sensitive and specific compared with other methods. Babies should be seen as early in life as possible, preferably in the first six months before pulmonary vascular disease can develop. Surveillance of recent laboratory records by the regional medical genetics service is an important adjunct, permitting the detection of new cases that are not referred by clinicians, and should be included in the screening process when possible.

We thank all the obstetricians and paediatricians who diligently referred their patients to us; the medical genetics laboratory staff and Jayne Rogers for their expert technical help; and Dr Mark Reid for his help and encouragement during this study.

1 Elwood JM, Darragh PM. Severe mental handicap in Northern Ireland. $\mathcal{F}$ Ment Defic Res 1981;25:147-55.

2 Rowe RD, Uchida IA. Cardiac malformations in mongolism: a prospective study of 184 mongoloid children. Am F Med 1961;31:726-35.

3 Cullum L, Liebman J. The association of congenital heart disease with Down's syndrome (Mongolism). Am f Cardiol 1969;24:354-7.

4 Shaher RM, Farina MA, Porter IH, Bishop M. Clinical aspects of congenital heart disease in mongolism. Am f Cardiol 1972;29:497-503.

5 Ades AE. Evaluating screening tests and screening programmes. Arch Dis Child 1990;65:792-5.

6 Tandon R, Edwards JE. Cardiac malformations associated with Down's syndrome. Circulation 1973;47:1349-55.

7 Park SC, Matthews RA, Zuberbuhler JR, Rowe RD, Neches WH, Lenox CC. Down syndrome with congenital heart malformation. Am f Dis Child 1977;131:29-33.

8 Gussenhoven EJ, Becker AE. Sequential segemental analysis in complex congenital heart disease: an echopathological correlation. In: Marcelleti $\mathrm{C}$, Anderson RH, Becker AE, Corno A, di Carlo D, Mazzera E, eds. Paediatric cardiology. Vol 6. Edinburgh: Churchill Livingstone, 1986:156-71.

9 Greenwood RD, Nadas AS. The clinical course of cardiac disease in Down's syndrome. Pediatrics 1976;58:893-7.

10 Sondon P, Stijns $M$, Tremouroux-Wattiej M, Vliers A. Precocity of pulmonary vascular obstruction in Down's syndrome. European fournal of Cardiology

11 Morray JP, MacGillivray R, Duker G. Increased perioperative risk following repair of congenital heart disease in Down's syndrome. Anesthesiology $1986 ; 65: 221-4$

12 Bull C, Rigby M, Shinebourne EA. Should management of complete atrioventricular canal defect be influenced by coexistant Down syndrome? Lancet 1985; ;:1147-9.

13 Wilson NJ, Gavalaki E, Newman CGH. Complete atrioventricular septal defect in the presence of Down syndrome. Lancet 1985;ii:834.

14 Johnson AM. Management of cardiac disease in Down's syndrome. Devel Med Child Neurol 1978;20:220-3.

15 Frontera-Izquerdo P, Cabezuelo-Huerta G. Natural and modified history of complete atrioventricular septal defect-a 17 year study. Arch Dis Child
1990;65:964-7.

16 Newfeld EA, Sher M, Paul MH, Nikaidoh H. Pulmonary vascular disease in complete atrioventricular canal. Am 7 Cardiol 1977;39:721-6.

(Accepted 4 April 1991) 